\title{
Prostate Cancer - Double Vision but Solitary Lesion
}

\author{
Lim HY ${ }^{*}$, Perchyonok $\mathrm{Y}^{2}$, Fitt $\mathrm{G}^{2}$, Lokan $\mathrm{J}^{3}$, Wada $\mathrm{M}^{4}$, Fabinyi $\mathrm{G}^{5}$, Azad $\mathrm{A}^{1}$, Cher $\mathrm{L}^{1}$ and Gan $\mathrm{HK}^{1}$ \\ ${ }^{1}$ Department of Medical Oncology, Austin Health, Heidelberg, Australia \\ ${ }^{2}$ Department of Radiology, Austin Health, Heidelberg, Australia \\ ${ }^{3}$ Department of Anatomical Pathology, Austin Health, Heidelberg, Australia \\ ${ }^{4}$ Radiation Oncology Centre, Austin Health, Heidelberg, Australia \\ ${ }^{5}$ Department of Neurosurgery, Austin Health, Heidelberg, Australia
}

${ }^{*}$ Corresponding author: Lim HY, Department of Medical Oncology, Austin Health, PO Box 5555, Heidelberg, Victoria 3084, Australia, Tel: +61 431175522 / +61 39496 5763, Fax: +61 39457 6698, E-mail: HuiYin.Lim@ austin.org.au

Citation: Lim HY, Perchyonok Y, Fitt G, Lokan J, Wada M, et al. (2014) Prostate Cancer - Double Vision but Solitary Lesion. J Case Rep Stud 2(2): 201. doi: 10.15744/2348-9820.1.501

Received Date: January 16, 2014 Accepted Date: April 28, 2014 Published Date: April 30, 2014

\begin{abstract}
A 53 year old man with a background of castrate-sensitive prostate cancer on intermittent androgen deprivation therapy (ADT) presented with right sixth nerve palsy secondary to a solitary right petroclival lesion involving adjacent dura and bone. The clinical and imaging characteristics of the lesion were consistent with a number of differential diagnoses (including metastatic prostate cancer, meningioma and chondrosarcoma). The patient initially declined biopsy and ADT was recommenced but the lesion continued to enlarge despite an excellent biochemical response. A subsequent biopsy of the petroclival mass demonstrated a WHO grade I meningioma and the patient proceeded to have definitive stereotactic radiotherapy. This case illustrates an unusual solitary skull base lesion in a man with prostate cancer. Whilst bony metastases, usually multiple, in the skeleton are common, solitary skull or brain lesions should be investigated as alternate diagnoses are likely in such circumstance.
\end{abstract}

Keywords: Petroclival lesion; Prostate cancer; Meningioma; Metastasis

List of Abbreviations: ADT-Androgen deprivation therapy; CT-Computed tomography; FDG-avid PET-Fludeoxyglucoseavid positron emission tomography; PSA-Prostate specific antigen

\section{Introduction}

Prostate cancer is the most common cancer in Australian men and the third most common cause of cancer deaths, comprising $13 \%$ of all cancer deaths in Australian men [1]. One in five Australian men will develop prostate cancer by age 85 with 1 in 25 risk of dying from prostate cancer [1]. However, many cases are indolent and patients often die from competing risks. Whilst the risk of mortality is only 3\% in low risk patients, it increases in intermediate risk (7\%) and high risk disease (18\%) [2].

Approximately $90 \%$ of patients with metastatic prostate cancer develop bony metastases, which are characteristically multiple osteoblastic lesions to the axial skeleton [3]. Bone metastases to the skull (8\%) and skull base (1.7\%) are much less frequent [3,4]. Similarly, patients with parenchymal central nervous system disease are also rare, being reported in less than 1\% of patients [5], although the prognosis is usually very poor in such cases [5,6]. Where these cases are reported, most $(79 \%)$ involve the brain parenchyma itself although some (21\%) involve dura only [5]. Importantly, the finding of solitary metastasis to the central nervous system from prostate cancer is exceedingly rare [5].

Here, we report a patient with castrate-sensitive prostate cancer in biochemical remission who presented with progressive right sixth nerve palsy secondary to a solitary right petroclival lesion involving adjacent dura and bone.

\section{Case Report}

A 53-year-old man was referred by his primary physician for the management of an asymptomatic screen-detected prostate cancer, with a prostate specific antigen (PSA) level of 22.5 (institutional reference range $0.0-3.5 \mu \mathrm{g} / \mathrm{L}$ ). He subsequently underwent a radical prostatectomy and lymph node dissection in March 2010 which revealed a T3bN1 carcinoma (Gleason 4+5) with lymphovascular and perineural invasion, positive margins and extra-capsular extension in one of two involved lymph nodes. Post- 
operatively, his PSA nadir level was $4 \mu \mathrm{g} / \mathrm{L}$ but without evidence of macroscopic metastases. He was commenced on ADT with goserelin acetate for 6 months and achieved biochemical complete remission (PSA<0.01) in March 2011. However, treatment was poorly tolerated and an intermittent schedule was adopted.

In July 2011, the patient began experiencing mild diplopia on right gaze which progressed over several months. Examination findings were consistent with mild right-sided sixth nerve palsy. Investigations showed a $10 \mathrm{~mm}$ x $3 \mathrm{~mm}$ extra-axial, durallybased, homogenously enhancing mass arising from the petroclival ligament. There was evidence of bony invasion with lysis but no sclerosis (Figure 1a \& 1b). Concurrently, his PSA rose from $0.01 \mu \mathrm{g} / \mathrm{L}$ to $0.04 \mu \mathrm{g} / \mathrm{L}$ over 5 months but importantly, re-staging CT and bone scan showed no evidence of other visceral or bony disease. The lesion was only minimally FDG-avid on PET. The patient was offered a biopsy to differentiate between the differential diagnoses of a solitary prostate cancer metastasis or, more likely, an unrelated pathology such as meningioma or chondrosarcoma. The patient declined a biopsy and requested recommencement of ADT for presumed petroclival metastasis from prostate cancer.

With ADT, the patient's PSA fell steadily over the next 6 months but unfortunately, his diplopia worsened and the lesion continued to enlarge (Figure 1c \& 1d). He also developed subtle signs of right trigeminal nerve involvement, manifested by reduced corneal sensation. The patient agreed to a biopsy and limited decompression of the lesion. Histopathology demonstrated a grade 1 meningioma with an elevated ki67 index, without evidence of carcinoma (Figure 1e \& 1f). The patient underwent definitive stereotactic radiotherapy 50 Gy in 20 fractions prescribed to $90 \%$ isodose ( 5 fractions per week over 4 weeks), with stabilisation of his neurological deficits

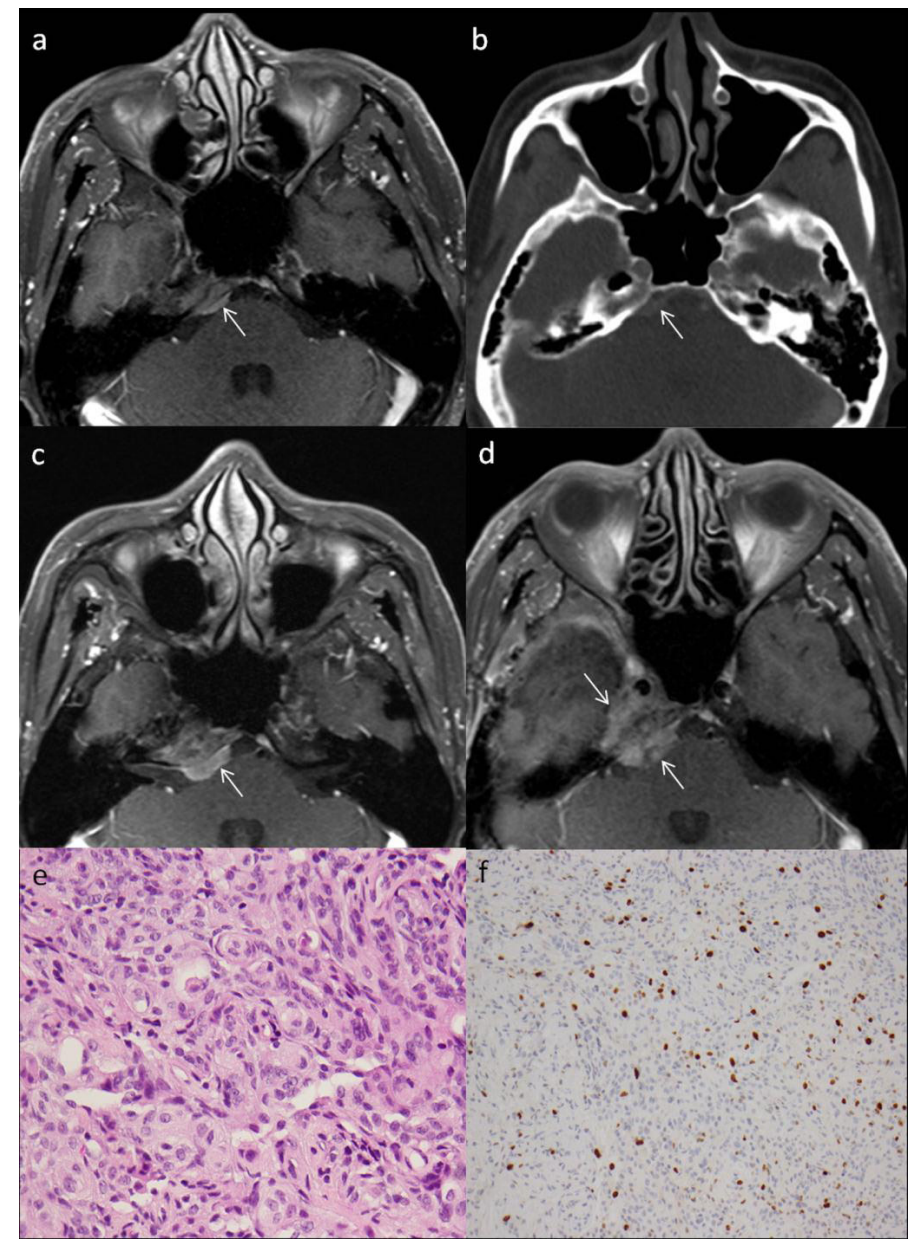

Figure 1: (a) Axial post contrast T1 MR. (b) Bone window CT images demonstrate a durally-based, homogeneously enhancing petroclival mass (arrow) with associated bone lysis when compared to the bone density on the left. (c) Corresponding MRI 10 months and (d) 18 months later with increase in lesion size and progressive bone invasion. (e) High power images (400x) showing characteristic morphological features of meningioma with syncytia, nests and whorls of meningothelial cells. (f) A ki67 stain of the lesion showing moderately elevated ki67 proliferative index.

\section{Discussion}

This case highlights the diagnostic challenge of a solitary base of skull lesion in a patient with prostate cancer presenting with a sixth nerve palsy. Although cases of diplopia from cranial nerve palsies [6,7] or ocular rectus muscle invasion [8] from metastatic prostate cancer have been reported, these are relatively rare. Whilst it would have been tempting to make a presumptive diagnosis 
of metastatic prostate cancer, a number of features in this case raised the possibility for an alternate diagnosis: the general rarity of solitary metastatic lesions in the brain or skull; the relatively low absolute levels of PSA; and the radiological appearance of the osteolytic focus in the petroclival region. It should be noted that a negative PSA does not exclude the presence of brain metastasis $[9,10]$. However, a diagnostic biopsy was initially declined by the patient on several occasions. The most common causes of clival lesions are primary tumours such as chordomas or chondrosarcomas (83\%) with the rest being meningiomas and metastatic disease from sites such as the lung, prostate, liver or skin (melanoma) [11].

Our patient was ultimately diagnosed and treated for a meningioma. The diagnosis of meningioma was considered in his initial differential diagnosis but the rarity of skull base meningiomas (2.8\%) [12] and the rapid rate of change argued for a diagnosis of metastatic disease, given that most meningiomas tend to remain asymptomatic and are slow growing (one series showed 35 of 57 patients displayed no growth over a 29 -month follow-up period) $[13,14]$. There is also sparse literature showing that metastatic prostate cancer can simulate meningiomas [15-17]. One intriguing possibility is the hypothesis that the unusual behaviour of the meningioma in this case may be the result of using luteinising hormone releasing hormone (LHRH) agonists as there is emerging data that some meningiomas may be regulated by the LHRH receptor expression [18-20].

\section{Conclusion}

This is a rare case of a concomitant atypical meningioma, possibly further stimulated by the use of ADT for prostate cancer treatment. Solitary lesions in the brain or skull of patients with a history of prostate cancer should be aggressively investigated given their rarity and the range of alternate diagnoses which can present similarly. The prognosis and management of many of these alternate diagnoses also differ substantially from those of metastatic prostate cancer and a high index of suspicion would aid an earlier diagnosis.

\section{References}

1) Australian Institute of Health and Welfare (2012) ACIM (Australian Cancer Incidence and Mortality), Canberra, Australia

2) Daskivich TJ, Fan KH, Koyama T, Albertsen PC, Goodman M, et al. (2013) Effect of age, tumor risk, and comorbidity on competing risks for survival in a U.S. population-based cohort of men with prostate cancer. Ann Intern Med 158: 709-17.

3) Bubendorf L, Schöpfer A, Wagner U, Sauter G, Moch H, et al. (2000) Metastatic patterns of prostate cancer: An autopsy study of 1589 patients. Hum Pathol 31: $578-83$.

4) Long MA, Husband JE (1999) Features of unusual metastases from prostate cancer. Br J Radiol 72: 933-41.

5) Tremont-Lukats IW, Bobustuc G, Lagos GK, Lolas K, Kyritsis AP, et al. (2003) Brain metastasis from prostate carcinoma: The M. D. Anderson Cancer Center experience. Cancer 98: 363-8.

6) McAvoy CE, Kamalarajab S, Best R, Rankin S, Bryars J, et al. (2002) Bilateral third and unilateral sixth nerve palsies as early signs of metastatic prostatic carcinoma. Eye 16: 749-53

7) Riemenschneider MJ, Beseoglu K, Hanggi D, Reifenberger G (2009) Prostate adenocarcinoma metastasis in the pituitary gland. Arch Neurol 66: 1036-7.

8) Leonard GD, Battiwalla M, Stockle JB, Harris RA, Monahan BP, et al. (2003) Extraocular muscle palsy from metastatic prostate cancer. Lancet Oncol 4: 358.

9) Fervenza FC, Wolanskyj AP, Eklund HE, Richardson RL (2000) Brain metastasis: an unusual complication from prostatic adenocarcinoma. Mayo Clin Proc 75: 79-82.

10) Salvati M, Frati A, Russo N, Brogna C, Piccirilli M, et al. (2005) Brain metastasis from prostate cancer: Report of 13 cases and critical analysis of the literature. J Exp Clin Cancer Res 24: 203-7.

11) Pallini R, Sabatino G, Doglietto F, Lauretti L, Fernandez E, et al. (2009) Clivus metastases: Report of seven patients and literature review. Acta Neurochir 151: 291-6.

12) Sade B, Chahlavi A, Krishnaney A, Nagel S, Choi E, et al. (2007) World Health Organization Grades II and III meningiomas are rare in the cranial base and spine. Neurosurgery 61: 1194-8

13) Radhakrishnan K, Mokri B, Parisi JE, O’Fallon WM, Sunku J, et al. (1995) The trends in incidence of primary brain tumors in the population of Rochester, Minnesota. Ann Neurol 37: 67-73.

14) Zeidman LA, Ankenbrandt WJ, Du H, Paleologos N, Vick NA (2008) Growth rate of non-operated meningiomas. J Neurol 255: 891-5.

15) Chen TY, Lee HJ, Wu TC, Tsui YK, Wu TC (2011) Intracranial dural metastatic prostate cancer can mimic meningioma: a report of two cases. Clin Imaging 35: 391-4.

16) Lath CO, Khanna PC, Gadewar S, Patkar DP (2005) Intracranial metastasis from prostatic adenocarcinoma simulating a meningioma. Australas Radiol 49: 497-500.

17) Lyons MK, Drazkowski JF, Wong WW, Fitch TR, Nelson KD (2006) Metastatic prostate carcinoma mimicking meningioma: case report and review of the literature. Neurologist 12: 48-52.

18) van Groeninghen JC, Kiesel L, Winkler D, Zwirner M (1998) Effects of luteinising-hormone-releasing hormone on nervous-system tumours. Lancet 352: $372-3$.

19) Lee KL, Terris MK (2003) Luteinizing hormone-releasing hormone agonists and meningioma: a treatment dilemma. Urology $62: 351$

20) Hirota Y, Tachibana O, Uchiyama N, Hayashi Y, Nakada M, et al. (2009) Gonadotrophin-releasing hormone (GnRH) and its receptor in human meningiomas. Clin Neurol Neurosurg 111:127-33. 


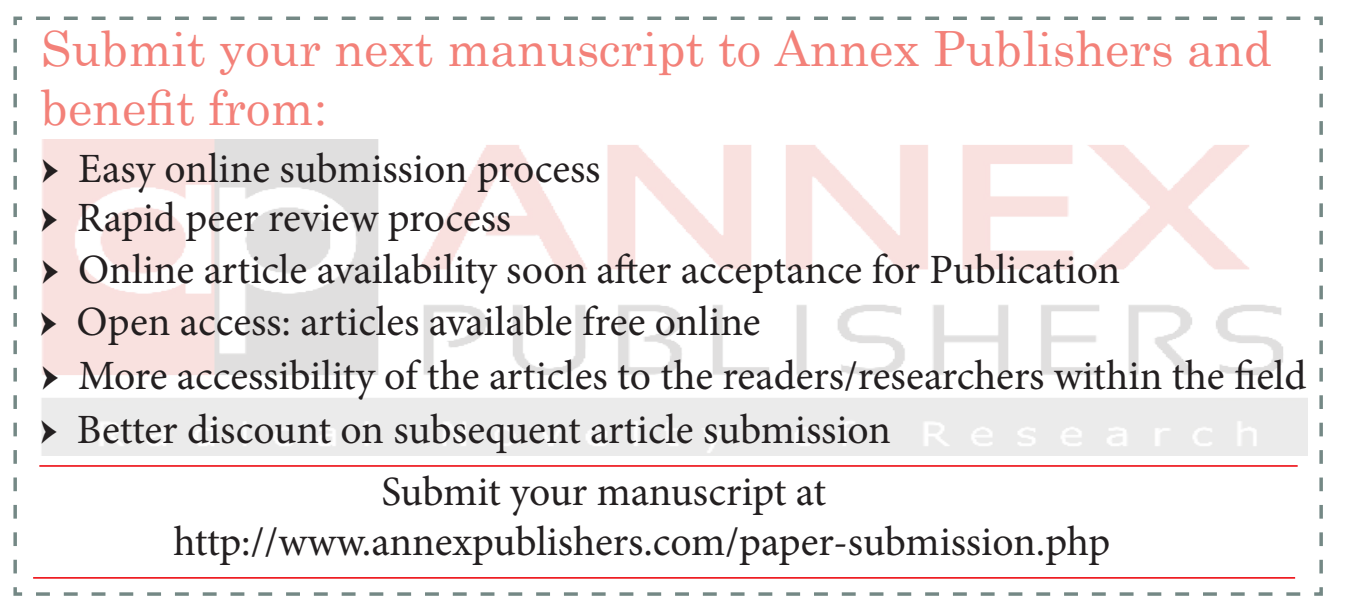

